\title{
Single-Molecule Analysis of Nucleotide-Dependent Substrate Binding by the Protein Unfoldase ClpA
}

Mary Farbman, Anne Gershenson, Stuart Licht

\section{Experimental Methods}

ClpA-FLAG and ClpA-K501R-FLAG Preparation. A pET9a plasmid containing the clpA gene was generously provided by Profs. Tania Baker and Robert Sauer. The FLAG sequence (DYKDDDDK) was appended to the $\mathrm{N}$-terminus of the gene using PCR amplification with the primers 5'-GGC GGC CAT ATG GAT TAC AAG GAT GAC GAC GAT AAG ATC CTC AAT CAA GAA CTG GAA C -3' and 5'-GGC GGC GGA TCC TTA ATG CGC TGC TTC CG -3'. A Stratagene Quikchange kit was used to generate FLAG-tagged ClpA K501R mutant plasmid; this construct was subjected to DNA sequencing to verify its identity. Plasmids were transformed into electrocompetent BL21(DE3)pLysS E. coli cells. Both the wildtype and mutant proteins were expressed and purified as described previously for wildtype ClpA (Choi and Licht 2005).

Cy3-ssrA Preparation. The eleven amino acid ssrA peptide AANDENYALAA was prepared by the MIT Biopolymers Laboratory. The peptide's purity and identity were confirmed by HPLC and mass spectrometry. The amino terminus of the peptide was labeled with Cy3-NHS ester (GE Healthcare Biosciences) and purified via rp-HPLC.

ATP $\gamma$ S Characterization. Adenosine-5'-O-(3-thiotriphosphate) was obtained from Roche Diagnostics as a tetralithium salt. The manufacturer's stated purity of the reagent was $\geq 90 \%$. To determine the extent of ADP contamination, we conducted a coupled enzyme assay whereby NADH oxidation, monitored by absorbance at $340 \mathrm{~nm}$, is coupled to ADP production by pyruvate kinase and lactate dehydrogenase. ATP $\gamma \mathrm{S}$ samples were found to contain $7 \% \mathrm{ADP}$ by this analysis.

TIRF Sample Preparation. Glass slides ( $24 * 60$ mm, No. 1 1/2, Electron Micrcoscopy Sciences) were cleaned by successive sonication treatments in $2 \%$ Micro-90 solution (1-2 hours), $100 \%$ ethanol (1-2 hours), and $0.1 \mathrm{M}$ potassium hydroxide (20 minutes). Slides were thoroughly rinsed with MilliQ water between each treatment and were stored in $100 \%$ ethanol until ready for use. Cleaned slides were dried with pressurized nitrogen, and 4-5 lanes were demarcated on each slide with silicone vacuum grease. Glass coverslips ( $22 * 22 \mathrm{~mm}$, No. 0, Electron Microscopy Sciences) were applied to the center of each slide. The resulting flow-cell lanes on each slide had a capacity of 25-30 microliters.

The bovine serum albumin, BSA, (Sigma, 98\% pure) used to block the slides was found to have catalytic quantities of proteases that degraded the BSA within minutes (data not shown). To prevent degradation, the BSA was treated with a general protease inhibitor cocktail (PIC) (Sigma P2714) for four hours at room temperature; small 
molecule inhibitors were separated from BSA by dialysis. Treated BSA was shown to be stable in solution for several hours.

To immobilize ClpA on the glass surface (Figure S1), slides were initially treated with a $0.5 \mathrm{mg} / \mathrm{mL}$ solution of AntiFLAG M2 monoclonal antibody (Stratagene) and were then blocked with a $10 \mathrm{mg} / \mathrm{mL}$ solution of PIC-treated BSA. A 30 microliter volume of sample consisting of $100 \mathrm{nM}$ ClpA-FLAG or ClpA K501R-FLAG and $3 \mathrm{mM} \mathrm{ATP} \gamma \mathrm{S}$ in wash buffer (50 mM HEPES pH 7.5, $600 \mathrm{mM} \mathrm{KCl,} 20 \mathrm{mM} \mathrm{MgCl}_{2}, 1 \mathrm{mg} / \mathrm{mL}$ PIC-treated BSA, and $10 \%$ glycerol) was applied to each lane. A 120 microliter volume of wash buffer containing $3 \mathrm{mM}$ ATP $\gamma \mathrm{S}$ was passed through each lane, followed by a 30 microliter "coat" volume consisting of $10 \mathrm{mM}$ ATP or $3 \mathrm{mM}$ ATP $\gamma \mathrm{S}, 5 \mathrm{nM}$ Cy3-ssrA, and an $\mathrm{O}_{2}$-scavanging system (1500 units/mL catalase, $0.215 \mathrm{mg} / \mathrm{mL}$ glucose oxidase, 4.5 $\mathrm{mg} / \mathrm{mL}$ glucose, and $1 \%(\mathrm{v} / \mathrm{v})$ beta-mercaptoethanol) in wash buffer.

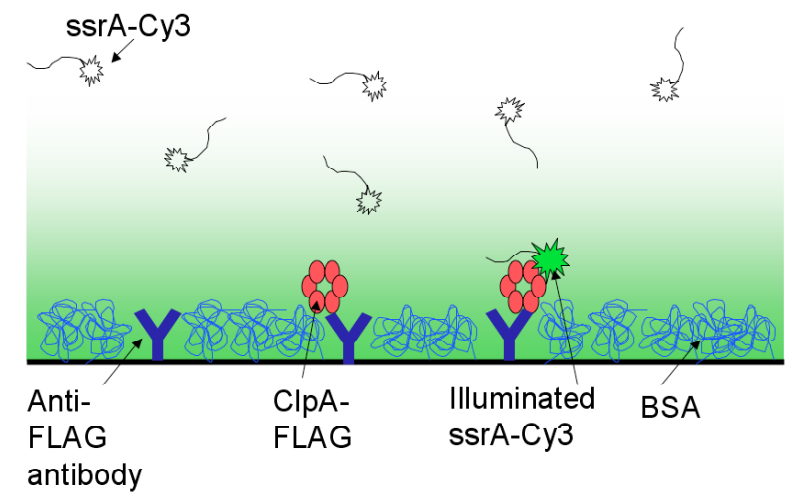

Figure S1. Schematic drawing of the experimental setup. ClpA-FLAG is bound to the glass surface via an anti-FLAG antibody. The substrate Cy3-ssrA is in solution above the surface along with ATP or ATP $\gamma \mathrm{S}$; when Cy3-ssrA molecules approach the surface, they fluoresce.

TIRF Instrument Set-Up and Measurement. Time on the imaging system used for data collection was generously provided by Professor Jeff Gelles at Brandeis University. The set-up (Friedman, Chung, and Gelles, Biophys. J. 2006, 91, 1023-1031) allows TIR excitation of fluorophores near the slide surface to be detected using an electron multiplying charge-coupled device camera. The intensity of the 532-nm line of an NdYAG laser was adjusted with neutral density filters; laser intensity immediately before the objective ranged from 2.11-2.15 milliwatts. Image acquisition software created in the Gelles lab (http://www.brandeis.edu/projects/gelleslab/glimpse/glimpse.html) was used to control data collection. Videos were collected at 36.0 $0.2 \mathrm{msec}$ per frame.

Analysis of TIR Fluorescence Data. Images were spatially bandpass filtered using a publicly available function written for Matlab (http://www.seas.harvard.edu/projects/weitzlab/matlab/latest_code/bpass.m ). A separate function, pkfnd (http://www.seas.harvard.edu/projects/weitzlab/matlab/latest_code/pkfnd.m), was used to 
locate areas of interest ("AOIs") with a threshold value of 70. AOIs measured 10 by 10 pixels, or $0.4 \mu \mathrm{m}^{2}$. Custom-written software created using Matlab (Mathworks) was used to track the intensity of detected spots throughout the frames of a given movie (Figure S2). For each AOI, a noise range determined from baseline values was entered by the user; intensities greater than the sum of the average noise plus 2.5 times the standard deviation of the noise was considered a fluorescent signal. The "on-time", or length of time in which the intensity consistently remained above the given threshold, was calculated for each AOI. Because of 1-2 frame "blinking" events, an off event was declared when the signal in 2 successive frames was below the signal threshold. For approximately $20 \%$ of the AOIs detected by the spot-picking function, the intensity was at or below the signal threshold in the twenty frames following the frame in which the AOI was identified, and these AOIs were omitted from the analysis.

Maximum likelihood fitting was used to obtain best-fit residence times from the observed on-times. The QuB software suite (Qin, Auerbach, and Sachs, Biophys. J. 1996, $70,264-280$ ) that was used for maximum likelihood fitting operates with time series datasets in which on-times alternate with off-times. Because only on-times were calculated from the data, it was necessary to construct time series in which experimental on-times were alternated with off-times drawn from a simulated distribution (an average off-time of $4 \mathrm{~s}$ was used). These datasets were analyzed using the maximum interval likelihood function of QUB. For each dataset, the data were fitted to Markov models with one or more "on" states and a single "off" state; the number of "on" states was increased until the inclusion of an additional state did not increase the likelihood of the fit. The simulated off-time of $4 \mathrm{~s}$ was recovered from the fit in all cases.
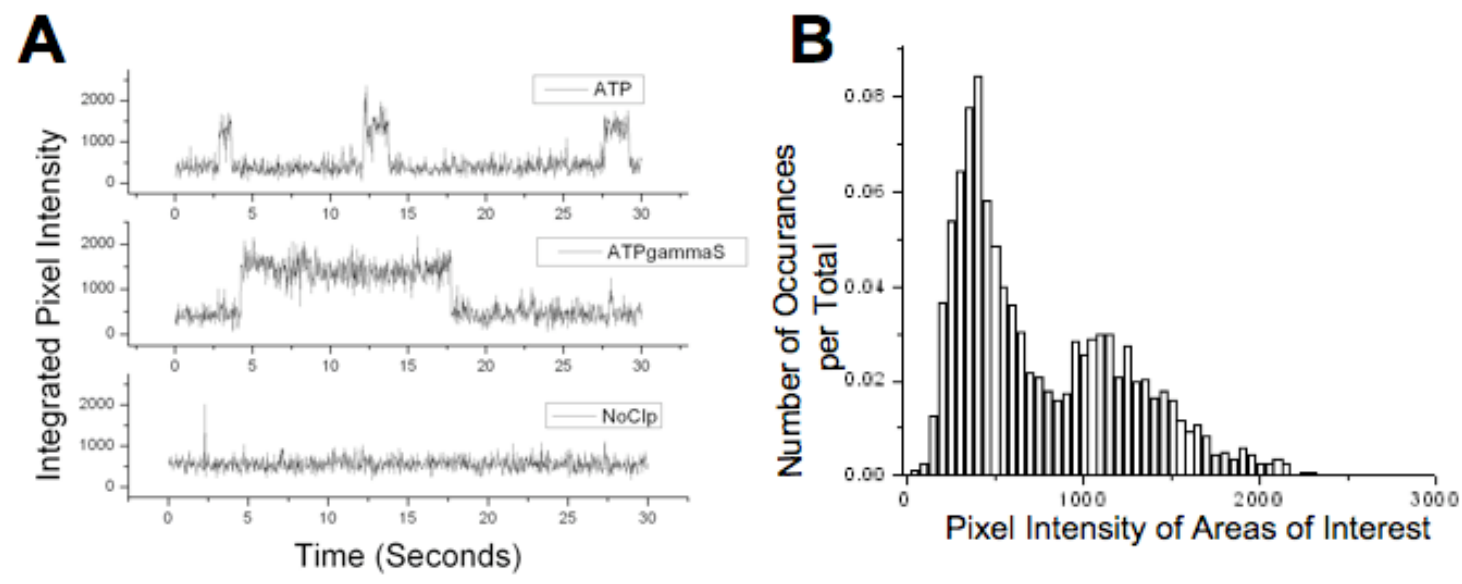

Figure S2. (A) Integrated pixel intensities over time for sample AOIs selected from movies taken in the presence of ATP and ClpA (top), ATP $\gamma \mathrm{S}$ and ATP (middle), and ATP but no ClpA (bottom). As is the case for other AOIs studied (data not shown), these sample plots demonstrate all-or-none gain and loss of fluorescence signals, suggesting single molecules are being observed. (B) Signal intensity histogram of data for WT/ATP condition. The distribution of intensities is well fit by a two-term Gaussian. The first, 
low-intensity term describes the signal when no substrate is bound; the second, higherintensity term describes the signal observed upon substrate binding.

SsrA Residence Time on CIpA K501R Mutant in the Presence of ATP $\gamma \mathrm{S}$. The distribution of substrate residence times on ClpA K501R in the presence of ATP $\gamma \mathrm{S}$ was found to be similar to that of ssrA residence times on the same mutant in the presence of ATP (Figure S3). Like the K501R/ATP distribution, the K501R/ATP $\gamma \mathrm{S}$ distribution is well-fit by a two-term exponential. The time constants for the distribution, as obtained from log-likelihood fitting, were found to be $190 \pm 40 \mathrm{~ms}$ and $1700 \pm 200 \mathrm{~ms}$; these time constants are within experimental error of those calculated from the K501R/ATP distribution.

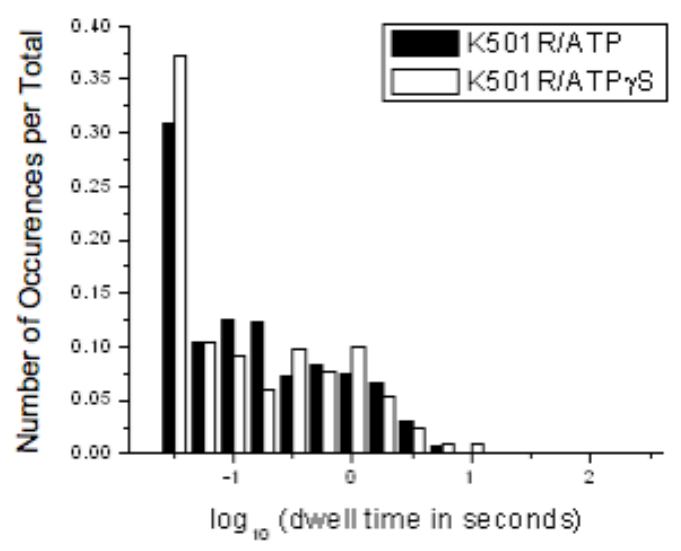

Figure S3. A comparison of the substrate residence times on K501R with ATP and with $\mathrm{ATP} \gamma \mathrm{S}$.

Independence of Distributions from Laser Power. The majority of our TIRF experiments were conducted using an NdYAG laser intensity of $2 \mathrm{~mW}$. Because photobleaching is power-dependent, we collected and analyzed data from the WT/ATP $\gamma \mathrm{S}$ condition (i.e., the condition yielding the longest residence times) at a lower laser intensity of $1 \mathrm{~mW}$. If photobleaching played a significant part in the observed distribution at $2 \mathrm{~mW}$, we would expect the fitting of the $1 \mathrm{~mW}$ distribution to yield longer residence times. Instead, we find a similar proportion of long-lifetime events in the two conditions. In particular, the fraction of events lasting longer than $10 \mathrm{~s}$ is $10 \%$ at $1 \mathrm{~mW}$ and $8 \%$ at $2 \mathrm{~mW}$. We conclude that substrate photobleaching does not play a significant role in the observed kinetics.

Association Rate Estimate. The relative association rates of Cy3-ssrA with the ClpAcoated glass surface for the different experimental conditions can be determined by plotting the cumulative number $n$ of AOIs that have been detected versus the time at which the $n$th AOI is detected. The slope of this curve represents two processes: (a) the rate at which the algorithm picks up spots missed from previous frames, and (b) the actual association rate of Cy3-ssrA molecules with the ClpA-covered surface. The former process dominates in the initial frames, where many spots present in the first 
frame are "detected" in the following two or three frames. The latter process becomes dominant in later frames, and therefore to compare the association rates between conditions, the slope of the curve was determined from the first ten AOIs landing after the initial five frames of illumination. The rates of association are approximately equal for the wild-type ClpA with ATP, K501R ClpA with ATP, and K501R ClpA with ATP $\gamma$ S (Figure S4). The rate of new spots appearing under the wild-type ClpA with ATP $\gamma \mathrm{S}$ condition, though, is about half the rate of the other three conditions, for reasons that are not yet clear. The association rate for Cy3-ssrA with wild-type ClpA in the presence of ADP, or with the surface in the absence of ClpA, is approximately $10 \%$ of the rate for wild-type ClpA with ATP.

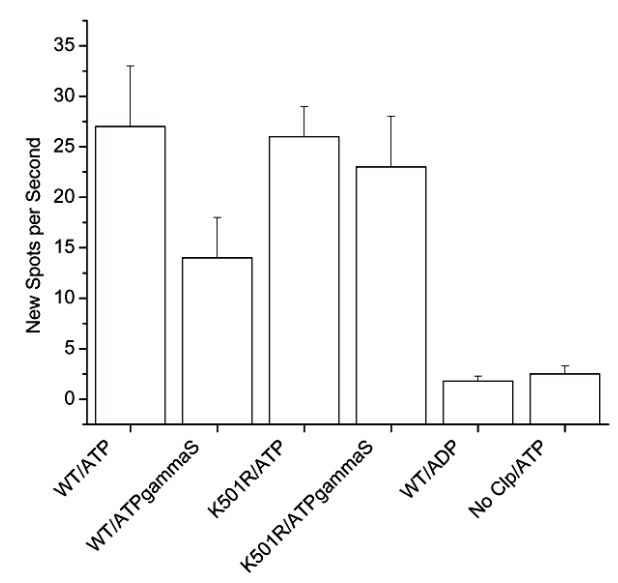

Figure S4. The rate of new spots appearing in frames. Uncertainties shown are the standard errors of the mean.

Steady-State Kinetic Assays. ClpA (50 $\mu \mathrm{M})$ was added to a $37^{\circ} \mathrm{C}$ mixture containing 25 $\mu \mathrm{M}$ ClpP, $10 \mathrm{mM}$ ATP, $50 \mathrm{mM}$ HEPES pH 7.5, $300 \mathrm{mM} \mathrm{KCl}, 20 \mathrm{mM} \mathrm{MgCl}{ }_{2}, 0.5 \mathrm{mM}$ DTT, $10 \%$ glycerol,102.8 mM aminomethylcoumarin (AMC, used as an internal standard) (I got rid of a space before AMC), and varying concentrations of Cy3-ssrA. Small samples were removed from the reaction mixture at various time intervals and mixed with guanidinium hydrochloride to a final denaturant concentration of $3 \mathrm{M}$; this concentration is sufficient to arrest ClpAP substrate degradation (data not shown). Samples were analyzed by $\mathrm{C} 4$ reverse phase chromatography. Enzymatic rates were calculated based on the integration of the major product peak with respect to the AMC internal standard. The enzyme displays Michaelis-Menten kinetics in its degradation of Cy3-ssrA (Figure S5) with a turnover number of $18 \mathrm{~min}^{-1}$ and a $\mathrm{K}_{\mathrm{M}}$ value of $7 \mu \mathrm{M}$. These values are similar to the steady state kinetic parameters for ClpA processing of ssrA-tagged protein substrates such as GFPssrA, which displays a turnover number of 15 $\min ^{-1}$ and a $\mathrm{K}_{\mathrm{M}}$ value of $5 \mu \mathrm{M}$ (Choi and Licht, Biochemistry 2005, 44, 13921-13931). 


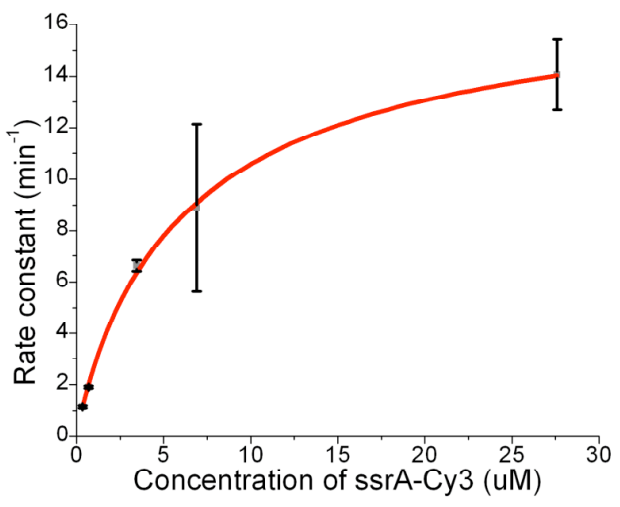

Figure S5. Michaelis-Menten plot of ClpAP degradation of Cy3-ssrA.

\section{ATP $\gamma$ S steady-state kinetic assays.}

A pyruvate kinase/lactate dehydrogenase coupled-enzyme assay was used to determine the rate of hydrolysis of ATP and ATP $\gamma \mathrm{S}$ by ClpA-FLAG as previously described (Choi and Licht, Biochemistry 2005, 44, 13921-13931). All reagents for this assay were purchased from Sigma. Reactions were performed with $7.5 \mathrm{mM}$ phosphoenolpyruvate, $0.2 \mathrm{mM}$ NADH, 19 units $/ \mathrm{mL}$ pyruvate kinase, 21 units $/ \mathrm{mL}$ lactate dehydrogenase, and $2.5 \mathrm{mM}$ nucleotide (ATP or ATP $\gamma \mathrm{S}$ ). $\mathrm{ClpA}_{6}$ was present at $60 \mathrm{nM}$ for assays containing ATP and $180 \mathrm{nM}$ for assays containing ATP $\gamma$ S. The hydrolysis of ATP by ClpA-FLAG was found to be $0.99 \pm 0.05$ micromoles ADP formed $/ \mathrm{min} / \mathrm{mg}$ ClpA, whereas the corresponding rate for $\mathrm{ATP} \gamma \mathrm{S}$ was measured at $0.019 \pm 0.01$ micromoles ADP formed $/ \mathrm{min} / \mathrm{mg} \mathrm{ClpA}$; thus the apparent hydrolysis rate for ATP $\gamma \mathrm{S}$ is approximately $2 \%$ of that for ATP. In using the same assay in the absence of ClpA, we determined the ADP contamination level of ATP $\gamma \mathrm{S}$ to be $6.9 \%$, which matched the level reported by the manufacturer. The coupled enzyme system converts ADP to ATP, and thus $6.9 \%$ of the ATP $\gamma$ S added to the assay is effectively converted to ATP that may be hydrolyzed by ClpA. Indeed, in the presence of $0.1725 \mathrm{mM}$ ATP (i.e., $6.9 \%$ of the ATP $\gamma \mathrm{S}$ concentration used for the original assays) ADP is formed at the rate $0.043 \pm 0.020$ micromoles $/ \mathrm{min} / \mathrm{mg} \mathrm{ClpA}$, or $4 \%$ of the ATP hydrolysis rate in the presence of saturating ATP. Thus the observed $2 \%$ rate of ATP $\gamma$ S hydrolysis appears to be due to ADP contamination of the ATP $\gamma \mathrm{S}$ sample rather than actual hydrolysis of the ATP $\gamma \mathrm{S}$ molecule.

\section{Simulations of rapidly equilibrating high- and low-affinity states.}

Rapid exchange between high- and low-affinity states would produce a system exhibiting only a single characteristic residence time. In contrast to results observed for NMR lineshapes, however, that residence time would not be the average of the interconverting species' residence times. Rather, in the limit of rapid interconversion between states, the 
residence time would approach a value close to that of shorter-lived state, because exits from this state would dominate the kinetics.

Simulations of dissociation from slowly- and rapidly-equilibrating states illustrate this effect. For slow interconversion $\left(5 \mathrm{~s}^{-1}\right)$ between states that dissociate rapidly $\left(1000 \mathrm{~s}^{-1}\right)$ and less rapidly $\left(100 \mathrm{~s}^{-1}\right)$, the residence time distribution has two characteristic times: 1 $\mathrm{ms}$ and $10 \mathrm{~ms}$. When the two states interconvert rapidly $\left(\sim 10,000 \mathrm{~s}^{-1}\right)$, the residence time shifts to $1.9 \mathrm{~ms}$. The difference of a factor of $\sim 2$ between this residence time and the short residence time in the slow exchange limit is a statistical factor that arises from the existence of two bound states; the same residence time is observed when the second bound state cannot dissociate at all.

A

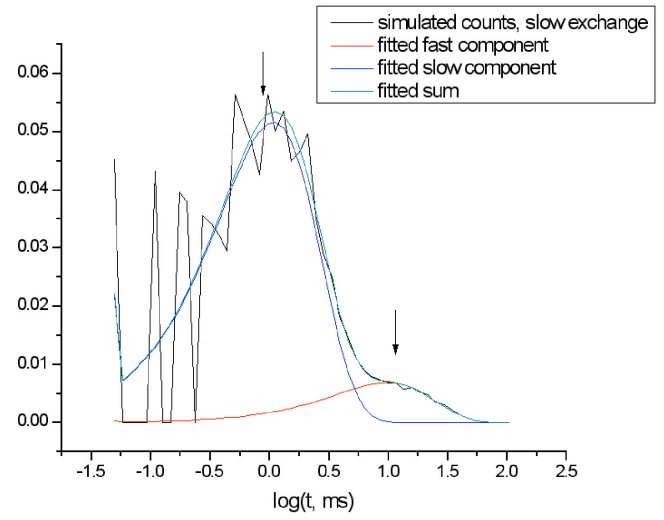

B

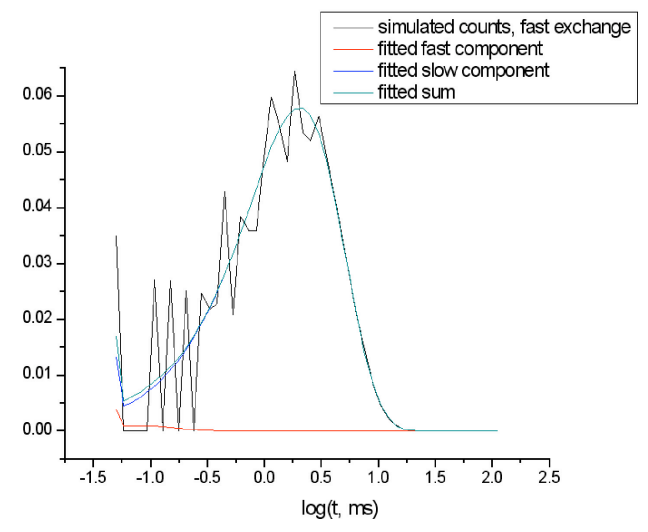

Figure S6. Simulations of slowly- and rapidly-equilibrating high- and low-affinity states. (A) Slowly-equilibrating states; the two distinct residence times are denoted with arrows. (B) Rapidly-equilibrating states. 
S-8 